\title{
À propos du livre de Gilles Labelle: Lécart absolu: Miguel Abensour
}

Gilles Labelle. Éditions Sens et Tonka, 2018, 382 pages. ISBN 9782845342774

Dr. Patrice Vermeren ${ }^{1}$

Je partirai du titre du livre de Gilles Labelle: L'écart absolu: Miguel Abensour, pour le rapprocher d'un propos tenu par Miguel Abensour dans l'entretien qu'il avait accordé à Manuel Cervera-Marzal et Nicolas Poirier en 2015: «Dans le cas du fouriérisme, les disciples ont clairement amoindri la nouveauté et l'écart absolu de Fourier, qui est devenu un écart tout à fait relatif. D'autant plus qu'il y a eu des jonctions avec des mouvements chré-

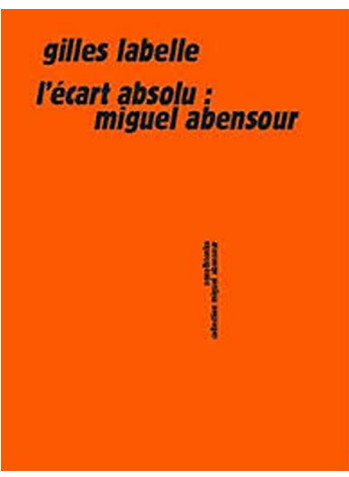
tiens. Dans mon article «Pierre Leroux et l'utopie», j'ai donc essayé de montrer qu'en général les véritables disciples sont les hérétiques et non les orthodoxes qui, au fond, ont pour seule ambition de ramener l'hétérogène dans l'homogène»².

L'écart est un terme de jeu: ce sont les cartes dont un joueur se défait. C'est aussi selon le dictionnaire de Littré l'action de s'écarter de sa direction, de se jeter de côté. L'écart absolu est un concept nodal chez Charles Fourier, qui écrit dans La Fausse industrie (1835): «tous les écrivains sont en quête d'un sujet neuf: la plupart, faute de nouveauté rée-

\footnotetext{
Dr. en Filosofía. Académico del Departamento de Filosofía de la Université Paris 8 Vincennes - Saint-Denis. Contacto: vermeren.patrice@gmail.com ORCID: https://orcid.org/0000-0002-5976-4393

2 «Entretien avec Miguel Abensour: «L'utopie et la lutte des hommes », sous la direction de Manuel Cervera-Marzal et Nicolas Poirier: Désir d'utopie. Politique et émancipation chez Miguel Abensour, Paris, L'Harmattan, 2018, page 17.
} 
lle, n'ont de ressource que l'esprit de contradiction: Aristippe contredit Chrysippe, Zénon contredit Epicure. Un débutant un peu adroit réussit à se faire remarquer, en prêchant l'opposé des opinions admises, en contredisant tout dans les conférences et les pamphlets». Mais Fourier use aussi du concept d'écart absolu en l'important de la statistique et prend l'exemple de Vasco de Gama et de Christophe Colomb: on ne découvre jamais rien en suivant les chemins déjà parcourus. Un exemple repris par André Breton dans le Manifeste du surréalisme (1929): «Il fallût que Colomb partît avec des fous pour découvrir l'Amérique», et un signifiant qui nomme la dernière exposition internationale du surréalisme à la Galerie de l'œil à Paris, en 1965, qu'André Breton a placé sous l'exergue de cette citation de la Théorie des quatre mouvements de Charles Fourier: «J'avais présumé que le plus sûr moyen d'arriver à des découvertes utiles, c'était de s'éloigner en tous sens des routes suivies par les sciences incertaines, qui n'avaient jamais fait la moindre invention utile au corps social; et qui, malgré les immenses progrès de l'industrie, n'avaient pas même réussi à prévenir l'indigence: je pris donc à tâche de me tenir constamment en opposition à ces sciences» ${ }^{3}$. Miguel Abensour, qui évoque André Breton reprenant le mot de Charles Fourier, serait donc placé lui-même selon Gilles Labelle dans cette voie oblique de la connaissance qui, comme Christophe Colomb selon Fourier, s'engage dans un Océan vierge, sans tenir compte des frayeurs de son siècle, et comme Breton essayant un mécanisme en contraste avec le nôtre, procéderait par écart absolu.

La question devient donc: de quoi Abensour et son œuvre sontils le nom, sous l'impératif de l'écart absolu? D'emblée le livre s'ouvre sur la taxinomie des points sur lesquels Miguel Abensour se sépare de Marcel Gauchet. Au premier abord, je n'avais pas saisi la pertinence du choix de Gauchet comme singe sommeillant à l'ombre d'Abensour, au sens où Nietzsche parle du singe de Zarathoustra. La lecture du livre de Gilles Labelle aura éclairé ma lanterne. Contre l'écart sans autre ou sans altérité, paradoxalement déployé au sein du Même, de Marcel Gauchet, la position «révoltiste» absolue de Miguel Abensour restitue la différence de l'instituant et de l'institué, la distinction du politique et

Charles Fourier: Théorie des quatre mouvements, 1808, p.7-8 ; voir Henri Béhar: «La méthode de l'écart absolu : Isidore Ducasse lecteur de Charles Fourier», Mélusine, 2014, 07. 
de la politique, la séparation des choses politiques -et de leur retourde la politique normale. Une différenciation que Gauchet s'est employé à occulter en donnant comme indépassable la visée individualiste et libérale du réel existant, et son recours à la Nécessité économique ou à la Morale pour mieux légitimer l'impossibilité et l'impensabilité d'un autre monde que celui de l'idéal de démocratie mondialisée des individus et du système matériel de la démocratie libérale du marché.

Notons qu'auparavant, il y avait eu d'abord entre Gauchet et Abensour une rencontre dans le sillage de Claude Lefort, sans doute à partir de l'exigence de réveiller la question de la philosophie politique, telle qu'elle apparaît sous la plume conjointe de Gauchet et de Lefort dans Textures en $1971^{4}$, où la question la plus centrale de toutes est donnée comme étant celle de la philosophie politique, ravivée dans l'idée de reconnaître que la philosophie politique relève de la pratique politique puisqu'elle est, dans la vérité de son mouvement, critique des idéologies, et requiert de porter un renversement de la posture traditionnelle d'être un discours clos, détaché de ce qu'il signifie, proféré depuis un lieu haut placé d'où le social et le politique pourraient faire figure de positivités susceptibles d'être séparées, hiérarchisées et envisagées ensemble ou successivement selon le point de vue de la théorie ou celui de la praxis. A cet égard, écrivent les auteurs, elle implique un renversement de la posture traditionnelle dans la mesure où, s'efforçant de porter l'interrogation au sein du politique, elle s'emploie à dévoiler que ce dernier renferme en soi l'interrogation sur son propre être, sa propre origine. Ce numéro de Textures contient deux études, l'une de Claude Lefort et Marcel Gauchet: «Sur la démocratie: le politique et l'institution du social», rédaction par Gauchet d'un cours de Lefort qu'il a suivi à l'Université de Caen en 1966-67, l'autre de Claude Lefort intitulée «La guerre, le discours sur la guerre et la politique», qui est une interprétation du Discours sur la Première Décade de Tite-Live sur Machiavel. Deux ans plus tard, Miguel Abensour commence à publier des extraits de sa thèse de doctorat ${ }^{5}$ dans Textures, et entre finalement en 1975 au comité de rédaction de la revue. En 1974, il rédige le manifeste de sa collection Critique de la politique chez Payot, se donnant

Textures $\mathrm{n}^{\circ} 2-3,1971$.

Miguel Abensour: «L'histoire de l'utopie et le destin de sa critique», Textures 6-7 1973 et 8/9 1974, repris dans Utopiques IV, Paris, Sens et Tonka, 2016. 
pour tâche de continuer l'héritage des années 60-70: résister aux entreprises de scientifisation ou de sociologisation du politique, mais en ayant réappris à l'épreuve de l'histoire -singulièrement mai 1968l'intelligence du politique, et à reconnaître dans le lieu du politique un foyer d'intelligibilité du social historique. La critique de Foucault et la lecture de Clastres sont sans doute deux éléments qui viennent conforter une redécouverte de la politique qui passe chez Lefort plutôt par une critique du totalitarisme, et se cristallise sur la réédition du Discours sur la servitude volontaire de La Boétie, en 1976, visant à compliquer la question machiavélienne, «comme si les deux noms symbolisaient le paradoxe du politique: Machiavel pensait le pouvoir avec la liberté, La Boétie pensait le pouvoir contre la liberté». Mais les chemins d'Abensour et de Gauchet vont bientôt diverger, comme si la filiation avec Lefort ouvrait l'horizon d'un fossé se creusant entre les disciples, les hérétiques et les orthodoxes, dont Martine Leibovici a décrit subtilement les méandres ${ }^{6}$, depuis le numéro de Passé-Présent sur la Terreur et l'article de Lefort sur Robespierre en 1983 jusqu'à sa publication de La complication, Retour sur le communisme en 1999. En tous cas, avec la publication du livre d'entretien avec Gauchet: La condition historique en 2003 et la réponse d' Abensour dans la revue anarchiste Réfractions, en 2004 à l'accusation de révoltisme portée contre lui, les couteaux sont tirés et Miguel Abensour persiste et signe: contre tout vouloir de la politique démocratique telle qu'elle existe et toute résignation à y participer sans réserve, comme si la démocratie pouvait être identifié au régime représentatif libéral et réduite à l'Etat de droit. Un écart qui se marque jusque dans les titres des livres: La Démocratie contre elle-même de Gauchet, titre d'un recueil paru en 2002, répond comme en écho à celui d'Abensour sorti en 1997: La démocratie contre l'Etat d'Abensour.

Et le choix de l'écart absolu relève de ce que Miguel Abensour nomme à propos de Clastres des «expérimentations interprétatives» qui produisent comme effets non la répétition des auteurs, mais de leur faire franchir un pas qui les disposera sur le terrain balisé par ce que Abensour désigne comme enquête sur les choses politiques, soit un \begin{tabular}{l}
\hline 6 Martine Leibovici : «Les institutions, les lois, la loi. Un bout de chemin avec Claude \\
Lefort», Désir d'utopie. Politique et émancipation chez Miguel Abensour, op.cit. p. 169.
\end{tabular} 
exercice proprement philosophique qui, loin de réifier les textes, leur restitue une contemporanéité, et par là, une vie. De là l'idée régulatrice de Gilles Labelle de procéder à l'égard d'Abensour de la manière dont il procède lui-même au regard des auteurs et des œuvres qu'il aborde, dans une infidèle fidélité, ou si l'on préfère une fidèle infidélité à Abensour lui-même. Faire du Abensour sur Abensour, mais on notera que Gilles Labelle ne va pas jusqu'à prétendre faire jouer Abensour contre Abensour. On sera attentif à la distance qu'il souhaite entretenir avec d'autres projets, celui d'une biographie intellectuelle dont il sait gré à Manuel Cervera-Marzal ${ }^{7}$ d'avoir épousé le genre honorable, ou celui de présenter une synthèse de ce que Abensour aurait vraiment dit, projet bien moins honorable qui ne ferait pas droit à un impensé de textes qu'il s'agit de rendre à leur inventivité. Et l'écart absolu de Miguel Abensour sera mis à l'épreuve d'un au-delà de trois concepts: le héros révolutionnaire comme paradoxe, l'utopie en tant qu'énigme et la philosophie politique critique comme tâche sinon inachevable, du moins inachevée.

Soit d'abord le choix du héros révolutionnaire comme personnage philosophique. Pour Labelle, l'inspiration de Miguel Abensour ne vient ni de Tocqueville, ni de Marx, mais plutôt de Michelet, et de l'énigme de la révolution à plusieurs. Question de Stimmung, dans un jeu complexe de tensions entre l'enthousiasme et l'effroi, le ravissement et la frayeur, que Gilles Labelle s'emploie à restituer selon quatre moments du parcours de Saint-Just: 1) le «cynisme plébéien» et le naturalisme, 2) le naturalisme en question, 3) la Révolution substituée à la nature et le héros révolutionnaire substitué à la révolution, 4) «héroïsme sans modèle», «institutions républicaines», et «suicide héroïque». Au terme de celui-ci, la question est celle d'un au-delà des tensions entre le possible de la liberté politique arrimant la pluralité à la quête du bien-vivre en commun et la réalité dans laquelle il s'embourbe, entre l'invention et l'imitation. Soit l'hypothèse d'une dialectique de l'émancipation où l'échec de la liberté, ou même le renversement en son contraire, à la-

Manuel Cervera Marzal : Miguel Abensour. Critique de la domination, pensée de l'émancipation, Paris, Sens et Tonka, 2013. Il revendiquait déjà dans cet ouvrage pionnier un travail avec plutôt que sur Abensour : «Plutôt qu'une présentation de son œuvre, il s'agit de l'investir pour entrer en sympathie avec le mouvement même de sa pensée » (p.13). 
quelle semblerait renvoyer le parcours paradoxal de Saint-Just selon Abensour, et Gilles Labelle identifie un élément crucial fourni par Abensour décrivant la posture induite par la Stimmung révolutionnaire et plus largement celle de la modernité politique: «Ne s'agit-il pas de rechercher, aussi bien dans les choses de l'amour que dans les choses de la politique, un point difficile à découvrir -peut-être même impossible à trouver- en tout cas instable et dont on ne saurait faire son séjour: un point qui garde quelque chose de l'héroïsme politique tout en nous préservant d'une dérive vers le sublime -«se guérir du sublime», écrit quelque part Abensour- et qui se tienne tout autant à distance de la platitude et de l'ennui de ce que l'on appelle, (...la «bourgeoisification de la vie», orientée vers la sécurité et la survie». («M. A.: La disposition hérö̈que et son aliénation», p.60-61), (qui est d'ailleurs noté «le dispositif héroïque» note 153, et on se demande pourquoi Gilles Labelle a-t-il enlevé la référence à Hobbes dans la phrase «ce que l'on appelle précisément en référence à Hobbes la «bourgeoisification» de la vie»?). En tous cas, la manière dont Gilles Labelle travaille ce texte allusif et tente de donner un statut nodal à ce «point» dans le dispositif spéculatif de Miguel Abensour est sans doute emblématique de la fidélité infidèle qu'il revendique dans sa lecture de Miguel Abensour, rejoignant la description qu'en fait Anne Kupiec dans sa préface à $L e$ cœur de Brutus: mobiliser des angles de vision différents pour révéler la complication d'une question, quel qu'elle soit, à laquelle aucune réponse définitive ne peut être donnée, et accueillant ainsi l'indétermination du politique ${ }^{8}$ ». Déterminer un point auquel les choses de la politique sont censées s'arrimer tout autant que les «choses de l'amour» donne matière à revenir sur la question du rire dans le parcours héroïque de Saint-Just, à aborder celle de l' affrontement de la condamnation à mort dans l'héroïsme postrévolutionnaire de Julien Sorel, et à s'interroger sur l'héroïsme anti-héroïque de Blanqui. Sur ce dernier en particulier, Gilles Labelle développe une relecture du texte lu par Miguel Abensour -De l'éternité par les astres- qui la redouble mais est en même temps une analyse originale, aboutissant à montrer comment Blanqui vise à susciter le réveil: «Plutôt que de céder, s'avouant finalement vaincu, à la fantasmagorie bourgeoise d'une répétition éternelle, Blanqui vise-

Anne Kupiec : préface à Miguel Abensour, Le cœur de Brutus, Paris, Sens et Tonka 2019 p. 20. 
rait à susciter un choc chez le lecteur en l'installant dans une posture où il devrait s'imaginer habitant un univers configuré comme si le Même le gouvernait tout entier, un univers absurde et effrayant» (p.91). Et Gilles Labelle relève deux motifs: D'abord «Être éveillé consisterait à habiter le monde comme si la singularité des formes était constamment sur le point d'être avalée par la logique du Même, comme si, pour reprendre et pousser jusqu'au bout une intuition de Walter Benjamin, une «catastrophe en permanence» planait sur le monde, menaçant de l'engloutir dans un "temps homogène et libre», synonyme d'une aliénation achevée» (p.93). Ensuite l'héroïsme pourrait exiger une sobriété en accord avec l'expérience du deuil des formes immuables que fait l'humanité, «encore que l'on pourrait s'interroger: une vie menée comme si on se trouvait devant une «catastrophe en permanence» ne définit-elle pas un « état d'exception»? Soit un dandysme de Blanqui comme posture d'un certain nombre d'extravagants, minorité agissante d'une aristocratie organisée en société secrète, plutôt que du grand nombre? On remarquera l'usage répété $\mathrm{du}$ «comme si» dans l'analyse de Gilles Labelle, se faisant sur ce point l'écho de la langue de Miguel Abensour. Jacques Derrida, dans un autre contexte, donne au «comme si» le statut de répondre à une ou plusieurs des possibilités ciaprès: «Que faisons-nous quand nous disons «comme si»? demande Jacques Derrida. a) Nous nous abandonnons à l>arbitraire, au rêve, à limagination, à lhypothèse ou à lutopie -certes, mais pas seulement. b) Nous mettons en œuvre certains types de jugements comme le jugement réfléchissant de Kant, qui "fait trembler" l'opposition entre la nature et la liberté. c) Et en outre, dans l'université, les arts ou la littérature, nous produisons des “œuvres»».

"C'est cette troisième dimension du "comme si" qui intéresse particulièrement Derrida, c'est celle-là dont l'importance est, pour lui, "sans limite". De quoi s'agit-il? D'un événement, un événement véritable, digne de ce nom, qui fait irruption sans prévenir, sans s'inscrire dans aucun horizon ni anticipation possibles. Cet événement ressemble à un acte performatif (car l'acte performatif, lui aussi, est un "comme si»), une ressemblance que Derrida compare à lassociation kantienne entre la nécessité irréductible de la nature, d'une part, et d'autre part l'art, qui fait intervenir la liberté». 
«Une opposition est convoquée entre deux ordres (chez Kant entre l'art et la nature, et chez Derrida, entre la singularité de l'œuvre et le travail universitaire), mais en même temps cette opposition est troublée par ce qui a lieu (l'événement du "comme si"). Un ferment déconstructif intervient en excès des deux ordres ${ }^{9}$ ».

Pierre Macherey a aussi consacré un long développement sur ce comme si: «L'université sans condition, ce serait donc l'université placée sous le signe du «comme si», qui constitue à la fois sa matière et sa règle. Devenue elle-même un "comme si», un devoir être, elle est le lieu où s'efface, au moins pour un temps, celui imparti aux activités universitaires, l'alternative du virtuel et du réel, entre lesquels, par sa magie propre, elle effectue un passage continue ${ }^{10}{ }_{\%}$. Et il indique les possibilités de dérive offertes par la mise en avant du «comme si» dans l'analyse de Derrida, donc de l'attitude propre à la philosophie, en tant que ferment révolutionnaire de la transformation de la chose universitaire. Ne peut-on rapprocher cet usage du "comme si" chez Gilles Labelle, dans sa lecture de Miguel Abensour, de celui qu'en fait Derrida? Le fameux point sur lequel se tiendrait Blanqui, comme si le Même gouvernait le monde et la catastrophe était inévitable, n'estil pas justement ce qui lui permet d'échapper à la résignation sans espoir de la fantasmagorie de la répétition propre à la modernité (soit la projection dans le cosmos de l'enfer de l'éternel retour du Même, donné comme l'essence de la modernité), en préservant la possibilité de la rupture, du réveil de l'utopie débarrassée du mythe, et de la persistance de la révolution?

Gilles Labelle met ensuite l'écart absolu de Miguel Abensour à l'épreuve de l' au-delà d'un second concept: après le héros révolutionnaire comme paradoxe, comme personnage philosophique paradoxal, l'utopie en tant qu'énigme, c'est à dire de scène d'images ou de simulacres à la fois "capable de générer un espace littéraire-politique, fondé sur le commun et la pluralité, et de faire voir, par là-même, en quoi le réel s'en écarte, et ainsi "ce qui lui manque" pour atteindre au bien" (p.130). Le paradoxe, c'était une opinion contraire à l'opinion commu-

\footnotetext{
$9 \quad$ https://www.idixa.net/Pixa/pagixa-1110150901.html.

10 Pierre Macherey : «La profession de foi de Derrida », La Philosophie au sens large, séminaire du ler décembre 2009.
} 
ne, une chose qui va contre elle: soit le héros révolutionnaire sans modèle, contrairement au héros de l'Antiquité, et toute la question était de savoir comment au point crucial il se trouve confronté au risque d'incarner finalement un modèle; les institutions républicaines pour sortir de la Terreur, mais au risque de figer la révolution dans la loi; le discours suicidaire de Julien Sorel, héros post-révolutionnaire, à son procès, comme répétant le suicide héroïque de Saint-Just... L'énigme, c'est selon Littré "une définition de choses en termes obscurs, mais qui, tous réunis, désignent exclusivement leur objet et sont données à deviner. (...) Par extension: tout ce qui n'est pas facile à comprendre, à deviner au premier abord". Ici encore, le fil conducteur de la lecture de Gilles Labelle est celle du rapport -en l'occurrence de l'utopie- aux choses politiques. Posant la question: comment lire l'utopie de Thomas More?, c'est à dire: comment la lire autrement que les interprétations attestées -éternelles ou persistantes- de l'utopie, il en dresse un tableau très original, singulièrement par la mise en scène de deux lectures faites à partir de Léo Strauss, baptisées lecture faible et lecture forte. Là aussi Gilles Labelle se prend au jeu de faire du Abensour audelà d'Abensour, non pas comme Martial Guéroult pouvait répondre à la place de Descartes à des objections qui n'avaient pas été faites à celui-ci de son vivant, mais avec une toute autre visée: non pas restituer la structure conceptuelle du dispositif philosophique de Miguel Abensour, mais prolonger son geste d'une interrogation des textes à partir de la question du retour des choses politiques, en l'occurrence avec un Léo Strauss au-delà de Léo Strauss, en opposant son enseignement faible et son enseignement fort, ce dernier n'étant autre que la lecture que propose en propre Miguel Abensour de Thomas More, non pas imitateur de Platon, mais plutôt inventeur d'un mode nouveau de saisir les choses de la vie politique, à l'instar de son contemporain Machiavel.

Gilles Labelle explore ensuite les avatars de l'extraordinaire floraison utopique -l'expression est d'Abensour du splendide XIX ${ }^{\circ}$ sièclecette fois-ci l'expression est d'André Breton, dans La clé des champs (1953, citée par Abensour en exergue de son article sur «Pierre Leroux et l'utopie socialiste», en 1974) et fait immédiatement écho pour nous, par antithèse, au (Le) stupide XIXe siècle, exposé des insanités meurtrières qui se sont abattues sur la France depuis 130 ans, 1789-1919, de Léon Daudet, paru en 1923. Il reprend comme matrice les trois gen- 
res de l'utopie, le socialisme utopique, le néo-utopisme et le nouvel esprit utopique, mais en faisant le choix de privilégier à chaque fois une figure spécifique. Et celle-ci est toujours traitée comme si elle se présentait identique à elle-même dans toute l'œuvre de Miguel Abensour, laissant entendre que pour Gilles Labelle il n'y a pas d'évolution dans la pensée de celui-ci, depuis ses textes de jeunesse et sa thèse de doctorat, jusqu'à ses derniers livres, en passant par les articles d'abord dispersés mais qui repris et complétés ont fait la matière des ouvrages achevés. Entendons-nous: il n'y a pas pour Gilles Labelle un système abensourien restituable sous la forme d'un compendium philosophique, mais il y a une visée implicite qu'il rend plus ou moins explicite d'une pensée qui n'a pas d'histoire, au sens où elle serait à contextualiser au regard du champ agonistique des penseurs du temps présent. En ce sens, peut-on prétendre qu'il y a unité de la pensée d'Abensour, comme si elle était commandée par une intuition de départ dont elle ne se serait jamais éloignée, mais qu'il se serait appliqué à déployer et à vérifier, par adjonction de nouvelles lectures et sous l'effet des circonstances du temps? L'un des éléments de cette intuition originaire aura été, j'y reviens, cette attention portée au “splendide XIX ${ }^{\circ}$ siècle". Ce n'est pas un siècle philosophique à la mode à l'époque lorsqu'Abensour entreprend ses premières recherches doctorales. Dans les années 1960, le discours des utopistes dont il parle est disqualifié comme idéologique par les althussériens, et il assigne à sa découverte de William Morris et à sa lecture du chapitre VII de Eros et civilisation de Marcuse, publié en 1963, de "s'être intéressé à la faculté de l'imagination à libérer la réalité historique et à l'orienter vers des formes de liberté et de bonheur appartenant à un principe de civilisation d'où aurait disparu la surrépression" ${ }^{11}$. Il faudra attendre mai 68 pour que Rancière et le collectif des Révoltes logiques fassent droit aux prolétaires saint-simoniens ou aux professeurs de philosophie qui leur sont contemporains saisis ou désaisis par l'Etat. Avant tout le monde, encouragé par Deleuze mais celui-ci ne le suit pas sur la question de l'utopie, Abensour aura fait accéder le dix-neuvième siècle, et singulièrement le XIX ${ }^{\circ}$ siècle français, à la dignité d'objet philosophique. Mais de quel XIX ${ }^{\circ}$ siècle et de quelle utopie s'agit-il?

11 Miguel Abensour: «Pourquoi la théorie critique?», Le souci du droit. Où en est la théorie critique?, Paris, Sens et Tonka, 2009 p.15. 
Gilles Labelle s'attache d'abord à la figure du socialisme scientifique, en privilégiant les saint-simoniens. Puisant son inspiration dans la lecture de Pierre Leroux, Miguel Abensour interroge le socialisme comme sortie de l'alternative entre le républicanisme jacobin et l'individualisme libéral: "il convient selon les premiers socialistes tout à la fois de faire sa part au surgissement de la masse des dépossédés sur la scène publique et politique (contre la stratégie libérale) et de rejeter l'idéalisme (contre la stratégie jacobine) en posant l'existence d'un lien entre l'expression de besoins et de désirs dont on peut localiser l'expression dans le grand nombre, et la volonté de bonheur" (p.141). Ce qui lui permet de confronter la figure du prophète de l'émancipation, Saint Simon, avec ses disciples proclamés, Saint Amand Bazard et Prosper Enfantin, et de poser la question de la démocratie religieuse au risque de se transformer en nouvelle théocratie. Gilles Labelle illustre ensuite la catégorie du socialisme scientifique avec Robert Owen, pour qui "l'entreprise capitaliste" devient la "matrice" de l'utopie et la société industrielle le lieu où l'institué et l'instituant, le monde objectif et la liberté, peuvent se féconder mutuellement dans une "vie de relations" fondée sur l'expressivité ou la réversibilité" (p.169). Pour déterminer la figure du socialisme utopique, Gilles Labelle puise d'abord dans la thèse de doctorat de Miguel Abensour, dont il découvre dans les articles postérieurs les prolongements et les échos d'une conversion utopique. Ensuite, la figure du néo-utopisme, "syncrétisme entre le socialisme utopique et les idées dominantes", effacement de l'écart absolu, tente de réconcilier le chimérique avec le monde existant, et permet de rendre compte du peu de cas que fait Abensour de Bellamy ou de Cabet. A la frontière entre les deux premières figures de l'utopie, le cas Fourier, que Gilles Labelle traite marginalement dans la note 495 page 170 (mais il faut voir qu'il y revient plus longuement ensuite). La troisième figure, le néo-utopisme, a droit au contraire à la plus grande attention. Retour à Blanqui, déjà évoqué à propos du paradoxe du héros révolutionnaire. Voici maintenant ce Janus biface confronté plutôt à l'énigme de l'utopie, et comme souvent chez Abensour, il y a l'idée que sa réputation sulfureuse le laisse méconnu dans l'opération de sauvetage qu'il opérerait du noyau valable de l'utopie, à l'instar de Marx. L'opération de sauvetage par transfert, on le sait, est un concept de Karl Korsch naturalisé abensourien dans sa thèse. Question d'écart de nouveau et toujours, puisqu'il s'agit pour l'idéologie dominante selon Blanqui de proscrire toute distance entre un mot et sa signification, confinant 
l'avenir du réel à la répétition de l'existant, et au contraire de préserver la proposition utopique comme propédeutique à la transformation sociale, révolutionnaire et socialiste, sous condition de comparaître devant le tribunal du mouvement réel de celle-ci. Après 1848, Blanqui serait celui qui s'emploie à condamner les plans de transformation sociale in abstracto, pour mieux opérer un sauvetage de l'utopie par transfert sur le terrain de la révolution. Comme Abensour le dira de Marx, Blanqui luiaussi ne critique pas l'utopie pour son excès, mais pour son manque de radicalité. Mais Marx va bien au-delà, dans la complexité d'une critique de Hegel mêlée à sa critique des utopistes, dans leur prétention à aller au réel historique pour se l'approprier, alors qu'ils confondent celui-ci avec les idées auxquelles ils prêtent la vertu illusoire de surmonter les tensions ou les contradictions qui déchirent le tissu social-historique (p.186, et Gilles Labelle cite La démocratie contre l'Etat: “c'est le propre de la métaphysique moderne que de subsumer le réel dans une idée"). Ce que Gilles Labelle résume dans une phrase, comme souvent chez lui, longue, précise et compliquée: "Il s'agit de "sauver" Hegel en quelque sorte contre lui-même, en opérant un "transfert" du noyau vrai de sa philosophie, qui tient en l'idée géniale mais qui reste abstraite d'une mobilité de l'être, sur le terrain découvert par "les Français modernes" d'une critique de la domination arrimée au tort fait à la classe la plus nombreuse et la plus pauvre -et en même temps de "sauver" ces derniers en quelque sorte également contre eux-mêmes, en opérant un "transfert" de leur découverte tout aussi géniale d'un rapport entre cette critique et l'établissement d'un lien social nouveau, mais qui se traduit dans des propositions qui font abstraction des mouvements réels de transformation de la société, sur le terrain balisé par Hegel d'un être défini par son historicité" (p.187). Gilles Labelle examine à partir de là trois moments de la critique marxienne de Hegel chez Abensour, passant d'un texte de Miguel Abensour à l'autre comme si l'écriture de La Démocratie contre l'Etat, Marx et le moment machiavélien, publié en 1997, était contemporaine de celle de Les Formes de l'utopie socialiste communiste, essai sur le communisme critique et l'utopie, daté de 1973. A l'horizon, une interrogation sur les conditions de possibilité d'utopianiser Marx, c'est à dire de le faire comparaitre devant l'utopie, et d'inverser la proposition de Lucien Goldmann: soit partir de Saint Simon pour comprendre Marx. Donc de creuser un écart absolu avec le réel existant, contre tous les marxismes, faisant rimer la prévision morphologique du communisme 
avec la prévision sympathique des utopistes, dans la sensibilité aux choses politiques "entendues comme mise en rapport d'un objet commun, le monde à transformer, et d'une pluralité d'êtres sensibles et parlants, qu'on la conçoive située dans la classe la plus nombreuse et la plus pauvre où loge le désir d'un ailleurs où pourrait prendre consistance la "volonté de bonheur" ou dans la classe prolétarienne dont l'état d'aliénation la motive à viser par-delà le capital l'établissement d'un rapport actif, vivant, avec le monde objectif" (p.226).

C'est ici que Gilles Labelle fait intervenir, après Saint-Simon, Fourier et Owen, le quatrième socialiste: Pierre Leroux, dont la posture singulière se revendiquerait comme autoréflexive d'une tradition philosophique qu'il s'agit de s'approprier en tant qu'elle vise l'émancipation, mais dont il faut s'écarter lorsque, par un mécanisme d'inversion, elle pourrait favoriser l'émergence de nouvelles formes de domination. Peut-être aucun écrit sur Abensour, jusqu'à aujourd'hui, n'a donné à Pierre Leroux cette place centrale que Gilles Labelle lui assigne (plus de trente pages), éclairant par là d'un jour nouveau une œuvre qui, par moment, aurait pu s'orienter vers la considération d'un Leroux contre Leroux, ayant réussi à rompre avec la métaphysique pour mieux s'orienter vers le seul "mouvement réel". Mais Gilles Labelle ne s'engagera pas sur cette voie qui le mènerait à un Abensour contre Abensour, se tenant fermement à le prolonger dans un au-delà de lui-même. On pourrait cependant s'interroger sur la manière dont Abensour aurait pu suivre son propre projet de reprendre les thèmes de la solidarité, de la réversibilité, et l'idée d'humanité chez Pierre Leroux, en rapport avec une philosophie de l'incarnation qu'il voulait confronter à une problématique phénoménologique, mettant en relation la pensée de Leroux et celle de Maine de Biran (il aura consacré une séance de son séminaire au Collège international de philosophie, dont le manuscrit a été égaré), et les réinterprétations récentes de cette œuvre, comme par exemple celle de Michel Henry ${ }^{12}$. Un des travaux les plus intéressants, à titre de prolégomène, serait de ce point de vue celui de Lucie Rey, montrant dans Les enjeux de l'histoire de la philosophie en France au XIX ${ }^{\circ}$

12 Miguel Abensour: «Philosophie et socialisme. Pierre Leroux ou du style barbare en philosophie», Cahiers du Collège international de philosophie, ${ }^{\circ} 1,1975$, repris dans Miguel Abensour: Utopiques 1. Le procès des maîtres rêveurs, Paris, Sens et Tonka, 2013, p.194. 
siècle. Pierre Leroux contre Victor Cousin (2012) comment Abensour fait accéder Leroux à la dignité de philosophe de l'Humanité instaurant une autre relation à l'universel que celle qui passe par l'Etat, (re)posant cette question de l'instauration philosophique en relation avec celle du style et du ton en philosophie, comme Abensour le fera lui-même au Centre de philosophie politique de l'Université de Reims ou au Collège International de Philosophie. Mais ce n'est pas la question de Gilles Labelle, pour qui la convocation de Pierre Leroux par Miguel Abensour est un enjeu majeur de la définition du nouvel esprit utopique, avec sa tentative de produire une philosophie de l'utopie.

La seconde convocation d'Abensour comme figure du nouvel esprit utopique retenue est William Morris. Ici encore Gilles Labelle fait preuve d'une attention minutieuse portée aux concepts qui orientent la sortie vers une confrontation avec les choses politiques, mais ce n'est plus l'humanité (comme Ailleurs et "expérience du monde, donnée dans le rapport de réversibilité ou d'échange généralisé entre partie et tout, entre pluralité et monde commun, qui s'auto-réfléchit" (p.238), et l'association (comme combinaison de l'éros, désir du bon et du bonheur, et de la philia, amitié sans fusion qui autorise la pluralité), comme chez Leroux; mais avec Morris une illustration de l'épochè utopique -et de son double mouvement de mise entre parenthèses et ouverture au tout autre- qui préserve l'utopie de tout fantasme ou mythe de la réconciliation pour faire de sa fragilité le lieu d'une expérimentation interminable et la forme de son inachèvement, avec toujours un règlement de compte avec Hegel, mais ici ce n'est plus en passant par Schelling et la religion, comme pour Pierre Leroux, mais par la fonction nouvelle de l'art devenu certes incapable de dire le sens de l'Absolu, mais pas pour autant voué à la gratuité, mais assurant l'annonce prophétique et collective d'un ailleurs incarnant "les promesses de bonheur, la vie bonne" sans nulle position de surplomb par rapport au social. Sur Abensour lisant Leroux et Morris, les propos de Gilles Labelle sont bien autre chose et beaucoup plus qu'un commentaire -au sens ou Michel Foucault dit qu'il s'agit, en énonçant ce qui a été dit, de redire ce qui n'a jamais été prononcé: “Commenter, c'est admettre par définition un excès du signifié sur le signifiant, un reste nécessairement non formulé de la pensée que le langage a laissé dans l'ombre, 
résidu qui en est l'essence elle-même, poussée hors de son secret; mais commenter suppose aussi que ce non-parlé dort dans la parole, et que par une surabondance propre au signifiant, on peut en l'interrogeant faire parler un contenu qui n'était pas explicitement signifié13". Ne peut-on dès lors prétendre que Gilles Labelle rejette le commentaire parce qu'il pense que Miguel Abensour a tout dit, mais que la richesse et la complexité de ses propos exigent d'être resitués et redistribués dans leur question régulatrice: comment l'annonce du retour des choses politiques peut-elle se tenir dans l'écart absolu de son recouvrement par le réel existant?

Paradoxe du héros révolutionnaire, énigme de l'utopie: Gilles Labelle peut dès lors aborder le troisième volet de son triptyque abensourien: la philosophie politique critico-utopique. Il part du constat de l'apparente extranéité de cette philosophie politique par rapport à toute discipline identifiée comme telle, de l'hétérogénéité des œuvres qui sont censées constituer sa tradition et de la disparité des thèmes qu'elle se donne comme objets. Cela lui permet de dresser un tableau, sinon de restituer un fil directeur, des lectures privilégiées de Miguel Abensour: Hannah Arendt, Claude Lefort, Emmanuel Lévinas, Walter Benjamin, T.W. Adorno, Jean-François Lyotard, Pierre Clastres, et de conclure sur La Boètie. Hétérogénéité des œuvres lues, qui renvoie à la disparité des objets constitués: le totalitarisme, l'élément humain et la démocratie sauvage, l'être rivé à l'Autre, les rêves du collectif et l'éveil, le choix du petit, l'intraitable, pour finir sur la servitude volontaire. Bien loin de tenter d'unifier les œuvres et d'identifier un caractère commun des objets mobilisés par la philosophie critico-utopique de Miguel Abensour, Gilles Labelle en travaille les tensions.

Qu'est-ce qu'une tension? Dans le Dictionnaire de philosophie de Christian Godin, on trouve quatre définitions: «1) au sens physique, état de ce qui est tendu (la tension d'une ficelle). Chez les Stoïciens, la tension (tonos en grec) est une force de cohésion maintenant les éléments dans leur unité; 2) au sens physiologique, ensemble des forces de contrainte internes auxquelles sont soumises les parois des artères et des vaisseaux sous l'influence de la pression du sang; 3) au sens

13 Michel Foucault: préface à la Naissance de la clinique, Paris, PUF, 1963 p. XII. 
psychologique (on dit aussi tension d'esprit), effort intense et soutenu de l'esprit. Concentration (au sens de Janet). Les Stoïciens usent du même mot de tonos pour désigner l'effort de lâme qui se raidit contre l'influence des choses extérieures; 4) Au sens social et politique, état critique de la situation pouvant déboucher sur la rupture (grève) ou sur la violence (guerre)». Où Miguel Abensour et Labelle après lui puisentils leur idée de souligner les tensions conceptuelles plutôt que les homogénéités dans les textes qu'ils convoquent? La réponse est incertaine, mais j'aurais volontiers formulé l'hypothèse que Miguel Abensour aurait pu rencontrer Gilles Deleuze sur ce point, un point qui cristallise toute la question de l'entre-deux. Mais je vois aussi que la tension est une catégorie familière de Gilles Labelle, qui écrit par exemple dans la présentation du numéro 16 de la revue Politique et sociétés datée de 1997 sur «La démocratie inachevée», en référant à Claude Lefort: «Le régime démocratique suppose des tensions ou contradictions qui appellent une constante interrogation de ceux et celles qui cherchent à penser ce qu'elle est ou ce qu'elle devrait être»; et aussi qu'il est l'auteur d'une contribution intitulée «Castoriadis et les tensions inhérentes à l'imaginaire politique», parue dans Cornélius Castoriadis. Réinventer l'autonomie, sous la direction de B. Bachofen, S. Elbaz et N. Poirier, Paris, éditions du Sandre, 2008.

L'infidèle fidélité, ou si l'on veut la fidélité infidèle du disciple hérétique est ici à son comble: non pas ramener le divers à l'un, comme tenterait de le faire un disciple orthodoxe qui chercherait à trouver chez Abensour la cohérence du dispositif spéculatif d'une philosophie de l'esprit utopique, mais repérer en tout et partout des écarts qui contribuent à la redécouverte des choses politiques, qui autorisent la prise en compte -en rupture avec la philosophie de l'utopie esquissée par le nouvel esprit utopique du dix-neuvième siècle- des phénomènes inédits qui apparaissent au vingtième siècle, singulièrement le totalitarisme et la dialectique qui transforme les mouvements d'émancipation en nouvelles formes de servitude. Soit se livrer à des expérimentations interprétatives à l'infini, susceptibles d'identifier des impulsions restituant à la liberté un sens politique, sans préjudice de leur convergence et sous l'emblème d'un signifiant chargé : celui de la constellation. Mais ici il ne s'agit pas d'astres voués à l'éternelle répétition de Même, mais de l'attention portée obstinément aux tensions mouvantes qui attestent du risque persistant de 
disparition des choses politiques, une attention renvoyée -le terme aussi est connoté, cette fois-ci du côté de Merleau-Ponty et de l'épochè phénoménologique- à l'étonnement comme condition de possibilité d'approche des choses politiques. Notons au passage la permanence dans les esquisses de philosophie politique critique de Miguel Abensour de la référence à la phénoménologie et à «une pratique radicale de l'épochè», de Lefort à Lévinas et de Adorno à Clastres. En témoignent par exemple les usages de la torsion que lui fait subir Lévinas, procédant à une inversion complète de l'égologie transcendantale et formulant l'extravagante hypothèse d'un humanisme qui ne trouve plus son socle dans le moi mais dans l'autre homme: «l'épochè phénoménologique telle que la pratique Lévinas ne se révèle-t-telle pas à terme, épochè utopique?» (p.323). En témoigne aussi la constitution, comme figure spectrale et négative, de Hobbes, définissant la norme par exclusion de ce qui n'est pas elle: le Contre Hobbes se retrouvant à chaque fois, de Lévinas à Clastres, en position d'être non pas défini positivement, mais comme un horizon d'émancipation toujours à (re)construire par négation de toutes les formes de résignation à la domination. On notera aussi les transitions ménagées par Gilles Labelle dans le passage d'un auteur à l'autre: le suivant lui permet toujours de souligner une tension nouvelle, par exemple: si c'est sur le fond d'une catastrophe que Walter Benjamin estime qu'il faut considérer les temps présents, ce n'est pas pour autant la seule ascension du national-socialisme, comme chez Lévinas, c'est aussi la faiblesse de la riposte théorique et praxique du mouvement ouvrier et socialiste et de l'impuissance des «rêves du collectif» à s'y opposer, qu'il s'agit de prendre en compte. Mais pour autant il faut tenir ensemble les deux bouts de la chaîne: soit l'image de l'éveil, ne pas céder sur le rêve, mais aussi considérer que l'humanité établit avec ses rêves un rapport d'aliénation, pour pouvoir permettre la naissance d'une subjectivité nouvelle. Encore une citation de Labelle pour attester de sa mise en scène de la complexité des tensions complexes qui forment la trame du dispositif spéculatif de la philosophie politique critique de Miguel Abensour: «Il y a en ce sens une véritable «énigme du lien humain», que rend visible le parcours de l'image onirique à l'image ambiguë, saturée de tensions et qui, éclatant, ouvre le passage à l'image dialectique -«énigme» en ce que le lien humain existe simultanément «assez» pour orienter vers lui, pour susciter l'éveil du 
dormeur prisonnier du cauchemar de l'aliénation, tout en n'existant «pas tant que ça», puisque sa consistance dépend de l'éveil même» (p.334).

Il faudrait encore évoquer les trois dernières références convoquées à l'appui de la philosophie politique critico-utopique: Adorno, qui repose avec le non-identique (le petit) la question du rapport d'Abensour à Hegel (un autre fil qui traverse le parcours abensourien de Gilles Labelle, susceptible de biens d'autres lectures), Lyotard, avec «l'intraitable» et «l'enfance», Pierre Clastres, accueillant le sauvage comme Autre, et théorisant la société contre l'Etat. Il n'est pas indifférent que Labelle fasse converger cette constellation de tension vers la question, destinée à demeurer question, de la servitude volontaire telle qu'elle est posée par La Boétie. Comme si la lecture du Discours sur la servitude volontaire était en elle-même une «expérience politique», «comme si le Discours sur la servitude volontaire était, dans sa texture même, la mise à l'épreuve du désir de liberté du lecteur, de chacun des tous uns» (M.A.: Du bon usage de l'hypothèse de la servitude volontaire). Gilles Labelle conclue sur un constat: la philosophie politique critico-utopique ne se propose pas de résoudre les tensions, et sur une question: pour mieux inviter à une expérience politique? Comme s'il s'agissait de se saisir des choses politiques dans un partage de leur interrogation en commun?

En tous cas, la lecture de Gilles Labelle ne cherche nullement à restituer le contexte de son écart absolu par rapport au champ agonistique de la philosophie contemporaine en France, jamais ne sont cités, par exemple, ni Althusser, ni les nouveaux philosophes. Il déploie et prolonge la pensée de Miguel Abensour au regard de la seule chose qui importe à ses yeux: les choses politiques et l'horizon de leur retour. Le livre de Gilles Labelle ne vise pas non plus à reconstituer un Abensour selon l'ordre de ses raisons, ou à produire une synthèse du système matériel de ses philosophèmes mis à l'épreuve de leur cohérence interne. Et en ceci il est bien un disciple plus que fidèle dans l'infidélité, ou infidèle dans la fidélité, de Miguel Abensour: Labelle aura poussé, plus avant que le philosophe de l'écart absolu lui-même, les conséquences de la citation emblématique de Feuerbach que Miguel Abensour replace en exergue de chacun de ses écrits: «Une philosophie qui n'est que l'enfant du besoin philosophique et une chose; 
mais une philosophie qui répond à un besoin de l'humanité est une toute autre chose ${ }^{14 "}$. philosophiques (1839-1845), trad. Louis Althusser, Paris, PUF, 1973, p.96. 